\title{
TARMED 1.04 wird auf den 1. April 2007 eingeführt
}

\author{
Das TARMED-Leitungsgremium hat am 5. Dezember 2006 beschlossen, die \\ neue TARMED-Version 1.04 am 1. April 2007 in Kraft zu setzen. Der neue \\ Browser und die zusätzlich notwendigen Dokumente, wie das Änderungs- \\ protokoll, sind seit dem 8. Januar 2007 auf der offiziellen Website www. \\ tarmedsuisse.ch verfügbar. Somit bleibt genügend Zeit für die Anpassungen \\ der Software.
}

\section{FMH Tarifdienst}

Korrespondenz: FMH Tarifdienst Gösgerstrasse 8 CH-4600 Olten Tel. 0622879696 Fax 0622879690 tarife@fmh.ch
Eine wesentliche Änderung in der TARMED-Version 1.04 ist die Einführung einer Dringlichkeitsinkonvenienzpauschale mit der Position Nr. 00.2505. Diese neue Tarifposition schafft die Möglichkeit, Leistungen abzurechnen, die ausserhalb der Praxisöffnungszeiten erbracht werden, aber keine echten Notfälle sind (Dringlichkeitsinkonvenienzpauschale $\mathrm{F}$ bei dringlichen Konsultationen/Besuchen ausserhalb der regulären Sprechstundenzeiten sowie Mo-Fr 19-22, Sa 12-19 und So 7-19 Uhr). Diese Leistungen konnten bis anhin nur unter «normalen» TARMEDPositionen abgerechnet werden. Es sei daran erinnert, dass Notfälle gemäss TARMED nur dann verrechenbar sind, wenn sich die Fachärztin / der Facharzt sofort und verzugslos um die Patientin / den Patienten kümmert.

\section{Dringlichkeitsinkonvenienz}

Die Dringlichkeitsinkonvenienzpauschale wird mit 45 Taxpunkten (TP) in den TARMED aufgenommen. Um die potentielle Volumenzunahme auszugleichen, mussten die Notfallinkonvenienzpauschalen angepasst werden:

\begin{tabular}{lrr} 
& \multicolumn{1}{c}{ alt } & neu \\
\hline Notfall A & 60 TP & 50 TP \\
\hline Notfall B & 120 TP & 80 TP \\
\hline Notfall C & 180 TP & 145 TP \\
\hline Dringlichkeit & 0 TP & 45 TP
\end{tabular}

Ohne diese Änderungen zur Wahrung der «Kostenneutralität» hätte die schon lange geforderte Dringlichkeitsinkonvenienzpauschale nicht eingeführt werden können.

Folgende Punkte sind weiter zu beachten:

1. Neben der kostenneutralen Einführung musste dem gesetzlichen Auftrag der Kostenträger
Rechnung getragen werden; nämlich, dass diese keine Entschädigungen übernehmen dürfen, die von anderen zu tragen sind. Dazu gehört unter anderem die Abgeltung der Präsenzzeit. Dieser Punkt ist bei der Gesundheitsdirektorenkonferenz (GDK) eingebracht worden und nun im Gespräch für mögliche Lösungsansätze.

2. Das erhöhte Inkassorisiko, das der Notfalldienst nach sich zieht, darf ebenfalls nicht von den Kostenträgern bezahlt werden. Hier gelten die entsprechenden kantonalen Bestimmungen nach Schweizer Obligationenrecht (OR).

3. Die Datenparität für die statistischen Auswertungen gegenüber den Kostenträgern ist durch die Aufbereitung und Auswertung der Rechnungsdaten der Leistungserbringer über die Trustcenter gewährleistet.

\section{Kommunikation}

Der Zeitpunkt der Einführung von TARMED 1.04 blieb lange unklar, da der Beschluss des Bundesrates auf sich warten liess. Eine vorgängige «offizielle» Kommunikation der Version 1.04 hätte zur Verunsicherung und einer allfälligen verfrühten Anwendung durch die Leistungserbringer geführt.

Auf der offiziellen Internetseite www.tarmed suisse.ch werden die Informationen jeweils veröffentlicht. Über die verschiedenen anderen Kommunikationskanäle wird erst nach der offiziellen Publikation informiert.

Die FMH hat sich bis kurz vor Einführung des TARMED dafür eingesetzt, dass die Notfallpauschalen, wie vereinbart, in Franken und nicht in Taxpunkten vergütet werden. Da diese Massnahme aber zur Absenkung der Starttax- 
punktwerte geführt hätte, hat man wieder auf diese Forderung verzichtet.

Eine Bereinigung der bestehenden Notfalldefinition im TARMED ist aus verschiedenen Gründen praktisch nicht möglich.

\section{Wie weiter?}

Die TARMED-Tarifpartner werden gemäss Bundesratsbeschluss aufgefordert, die Auswirkungen der neu eingeführten Dringlichkeitspauschale hinsichtlich Zielerreichung und Kostenentwick- lung bis Ende 2007 zu prüfen und allenfalls Änderungen vorzusehen.

Die Tarifpositionen betreffend letzte 5 Minuten wurden ursprünglich gemäss Intervention des Preisüberwachers in den TARMED aufgenommen. Verschiedene Möglichkeiten zum Ersatz werden mit den Schweizerischen Gesellschaften für Allgemeinmedizin (SGAM) und Innere Medizin (SGIM) diskutiert. Weitere Schritte sind geplant. 